\title{
Paul Ricoeur, Cinq études herméneutiques
}

Genève, Labor et Fides, coll. « Logos », 2013, 144 p., préface de Jean-Marc Tétaz.

\section{Benjamin Fabre}

\section{(2) OpenEdition}

\section{Journals}

Édition électronique

URL : http://journals.openedition.org/assr/28332

DOI : $10.4000 /$ assr.28332

ISSN : $1777-5825$

Éditeur

Éditions de l'EHESS

\section{Édition imprimée}

Date de publication : 31 décembre 2016

Pagination : 378

ISSN : 0335-5985

\section{Référence électronique}

Benjamin Fabre, «Paul Ricoeur, Cinq études herméneutiques », Archives de sciences sociales des religions [En ligne], 176 | octobre-décembre 2016, mis en ligne le 20 juillet 2017, consulté le 24 septembre 2020. URL : http://journals.openedition.org/assr/28332 ; DOI : https://doi.org/10.4000/ assr.28332

Ce document a été généré automatiquement le 24 septembre 2020.

(c) Archives de sciences sociales des religions 


\section{Paul Ricoeur, Cinq études herméneutiques}

Genève, Labor et Fides, coll. « Logos », 2013, 144 p., préface de Jean-Marc Tétaz.

\section{Benjamin Fabre}

\section{RÉFÉRENCE}

Paul Ricoeur, Cinq études herméneutiques, Genève, Labor et Fides, coll. « Logos », 2013, 144 p., préface de Jean-Marc Tétaz.

1 Nous avons lu Les aventures de la dialectique grâce à Merleau-Ponty. Lisons-nous dans ce recueil posthume de Paul Ricœur les aventures de l'herméneutique? De même que la dialectique sautait les barrières de la logique pour devenir l'armature d'une ontologie, pourrait-on dire qu'ici l'herméneutique effectue la même embardée et devient à son tour le principe d'une métaphysique ? La pensée méticuleuse de Ricœur cède-t-elle à un emballement qui peut laisser le lecteur déconcerté?

2 La première des cinq études rassemblées en 2013, mais déjà publiées entre 1975 et 1991 s'attache à déterminer la tâche essentielle de l'herméneutique. Ricœur évoque d'emblée un « problème » herméneutique. On peut se demander pourquoi une méthode assez universellement pratiquée poserait un " problème » avant d'avoir été présentée. De quoi s'agit-il ? «De l'interprétation des textes à partir des opérations basiques de la compréhension ». Ricœur déclare qu'« au niveau le plus élémentaire et le plus banal de la conversation il y a un travail d'interprétation». Chacun est ainsi chaque jour, herméneute sans le savoir, comme Monsieur Jourdain. Et ceci à cause de la polysémie, « ce trait des mots d'avoir plus d'une signification quand on les considère en dehors de leur usage dans un contexte déterminé ». La simple énonciation «il est sorti» peut recevoir une infinité de sens à partir d'un noyau élémentaire évoquant dans l'espace un certain mouvement. Qui est sorti ? Et d'où est-on sorti ? Et que veux dire ici sortir ? La réponse à ces questions sera le plus souvent immédiatement donnée par la situation où 
cet énoncé est émis. La polysémie, qui est aussi en quelque sorte une absence, se réduit aussitôt par le rôle sélectif du contexte. "La sensibilité au contexte », dit l'auteur, « est la contrepartie inéluctable de la polysémie ». En ce sens, rien de plus quotidien, en effet que l'herméneutique. Mais le contexte n'est pas toujours évident, il doit être déchiffré, s'il s'agit par exemple de saisir les intentions d'un locuteur qui peuvent être complexes, voire confuses. Pour cela, l'herméneutique peut exiger un travail et donc aussi des règles, une méthode, s'il s'agit d'interpréter correctement ce qui a été dit, et quand nous nous trouvons en face d'un texte, d'où l'auteur s'est évidemment retiré, discerner le message et doit même exiger un travail spécifique. Le locuteur n'est plus là, le contexte devient difficile à déterminer, il faut se fier souvent à de simples hypothèses.

Ricœur pose alors cette question: pourquoi l'herméneutique a-t-elle un rapport privilégié avec la question du langage? Comme s'il pouvait y avoir autre chose à interpréter que des signes, à moins d'étendre l'art d'interpréter aux nuages, ou aux entrailles de poulet, ce qui ouvrirait un tout autre type de méthode ou de recette. Si l'auteur pose cette question, c'est peut-être justement qu'il suppose, à ce moment, que tout est signe dans l'univers, et qu'ainsi l'herméneutique pourrait légitimement se présenter comme une ouverture métaphysique. Nous n'en sommes pas là dans la première étude, où Ricœur examine l'approche de l'herméneutique chez Schleiermacher et Dilthey. L'herméneutique se constitue peu à peu comme une science en ceci qu'elle considère les textes et les œuvres à travers lesquels un esprit s'est exprimé avec toute la rigueur et l'objectivité possibles, et comme une science " historique ", puisqu'elle permet "l'accession de l'individu à l'histoire universelle ", c'est-à-dire "l'universalisation de l'individu», en lui ouvrant la possibilité de « s'approprier » les mondes disparus par la compréhension de leurs œuvres. À partir de cette ouverture, nous dit Ricœur, Heidegger verra dans l'herméneutique non plus seulement une méthode rendant féconde la lecture du passé, mais l'expression « du mode d'être de cet être qui n'existe qu'en comprenant ». L'herméneutique se relie ainsi à la structure même de mon être. De méthode scientifique, elle devient la marque ontologique d'un être pour lequel comprendre c'est s'ouvrir au monde en anticipant « toujours déjà » ce qu'il en reçoit. La dimension épistémologique de l'herméneutique n'en est plus qu'un aspect secondaire. On peut même penser que la méthode herméneutique, en s'obligeant à une mise à distance de son objet pour mieux le comprendre, méconnaîtrait l'appartenance au devenir historique dont nous ne pouvons pas vraiment nous extraire.

4 C'est pourtant à la distanciation comme fonction essentielle de l'herméneutique que revient Ricœur dans le deuxième article du recueil. Il y refuse l'alternative apparue entre la mise à distance et l'appartenance, entre la méthode objectivante et l'« attitude ontologique », et voudrait la "dépasser ». On peut ici s'interroger : pourquoi y aurait-il une alternative à dépasser? Si l'on voit bien que la possibilité de comprendre l'autre, dans un texte ou une œuvre, s'enracine dans la possibilité humaine de faire sien, en quelque sorte, tout projet et de saisir le projet inclus dans tout objet, on ne voit pas pourquoi il y aurait à choisir entre un pouvoir, ontologique si l'on veut, et une méthode qu'il rendrait possible? Voir là une alternative et tenter de la dépasser pourrait conduire à la confusion de deux ordres que rien ne rend a priori incompatibles et finalement à ne voir plus dans l'herméneutique que le signe d'une transcendance enracinée dans le sujet humain. 
5 Ricœur affirme cependant la fonction «positive et productive » de la distanciation à partir de cinq thèmes qui en constituent la problématique : le langage comme discours, le discours comme une œuvre structurée, le passage de la parole à l'écriture, le discours comme projection d'un monde, et enfin le discours comme médiation de la compréhension de soi. Les quatre premiers thèmes constituent les fondements de la possibilité herméneutique : il s'agit bien de se trouver en face d'un monde proposé par le discours et tenu à une certaine distance afin de pouvoir le comprendre et ainsi se l'approprier. Pour l'auteur, la distanciation et l'appropriation se rapprochent étrangement. "Comprendre un texte, c'est aussi se comprendre devant le texte », c'est recevoir de lui une proposition de monde et d'existence : s'approprier, c'est alors se distancer de soi-même, se séparer des illusions du « sujet ». Une métamorphose ludique de l'ego. À ce moment, l'herméneutique épistémologique semble laissée de côté, et le texte suivant qui traite de l'identité dans ses rapports avec le récit ne nous y reconduit pas. C'est dire que l'alternative est moins dépassée que basculée, si l'on veut, vers une vision de l'herméneutique comme culture et formation de soi.

6 «Se connaître ", écrit Ricœur, c'est s'interpréter soi-même sous le double régime du récit historique et $\mathrm{du}$ récit de fiction. Ces récits sont les instruments d'une herméneutique du soi dont évidemment l'auteur a longuement étudié les voies dans d'autres ouvrages et particulièrement dans Soi-même comme un autre. Cette étrange formule veut écarter à la fois l'exaltation de la certitude absolue du cogito comme évidence de soi et l'humiliation nietzschéenne du moi incapable de sortir de lui-même, fût-ce pour s'affirmer. S'il y a une herméneutique du soi, c'est qu'il n'est pas le jeu, mais ce qui s'atteste précisément dans ce travail d'interprétation, attestation « qu'on appelle communément conscience morale", "assurance d'être soi-même agissant et souffrant » (Soi-même comme un autre, Paris, Seuil, 1990, p. 35) Si le soi s'objective dans une construction nourrie par les récits, il sera protégé des identifications illusoires ou mensongères qu'une herméneutique du soupçon devra écarter, et il ne trouvera une forme authentique qu'en se défendant de la catégorie de la substance et de la permanence par la médiation de l'autre, en se confrontant «à l'hypothèse de son propre néant [...] qui n'est pas le rien dont il n'y a rien à dire » (ibid., 196). À l'identité du même (idem), s'oppose l'identité ipse de la promesse tenue. Certes, ces belles analyses de Ricœur sont inséparables aujourd'hui de toute approche de la connaissance de soi. On voit que l'herméneutique, si elle conserve un souci d'objectivité et de distance contre tous les fantasmes de l'inauthentique devient en quelque sorte un exercice permanent de la réflexion critique où les problèmes de l'interprétation sont absorbés dans la perspective d'une injonction morale : deviens ce que tu es et que tu ne peux pas vraiment connaître sans manquer à toi-même.

7 Ricœur traite enfin de l'herméneutique biblique. La forme narrative propre au texte biblique ne peut pas se lier à n'importe quelle théologie, mais seulement à celle qui annonce JAHVÉ comme l'actant d'une histoire de délivrance. Il y a une théologie homogène à la structure narrative biblique. Certes. Mais s'il s'agit de dire que le dieu d'Israël n'est pas celui des Grecs, c'est bien évident; et s'il s'agit de penser que c'est ce dieu qui nous parle dans la Bible, comment le savoir autrement que par la foi, qui ne provient pas de l'interprétation du texte? Comment ne pas trouver étrange cette phrase de Ricœur : «c'est en allant jusqu'au bout d'une écoute de ce livre comme d'un livre parmi les autres, qu'on peut le rencontrer comme parole de Dieu »? S'il s'agit d'un livre "parmi les autres ", cela reste un livre, qui peut bien nous proposer l'image d'un 
certain Dieu au terme d'une analyse herméneutique, mais qui peut difficilement effectuer ce saut hors du livre, qui serait une rencontre effective du dieu qui a parlé là. Ricœur rencontre alors (p. 111) la critique des illusions, des arrière-mondes, à la façon de Marx, Nietzsche ou Freud : elle devient pour lui non plus une critique externe « du dehors", mais une critique interne qui appartient au travail de distanciation "que toute compréhension de soi devant le texte requiert». "Une herméneutique du soupçon fait aujourd'hui partie intégrante de toute appropriation du sens ». Louable souci de purification et d'authenticité, mais est-il si sûr que l'herméneutique du soupçon se laisse facilement mobiliser au service de la foi ?

8 L'élargissement de l'herméneutique devient patent dans le dernier texte du recueil. Ricœur entend montrer que la formule johannique "Dieu est amour" peut être interprétée comme une expansion de la formule hébraïque de l'Exode "Je suis celui qui suis » (Ex 3,14). Les circonstances dans lesquelles le dieu biblique se désigne ainsi incitent Ricœur à une méditation philosophique sur la portée ontologique de la formule: s'agit-il pour Moïse de souligner l'autorité du mandant? Ou d'une nonréponse aux questions de Moïse où l'« incognito » de dieu serait préservé ? La formule de Jean peut être prise dans la continuité du texte biblique : celui-ci suppose la position de Dieu comme sujet, et c'est de ce Dieu-sujet qui est maintenant appelé amour. Si Dieu est « un/seul », cette nouvelle attribution relance la formule : le dieu " qui requiert un amour exclusif est lui-même cet amour exclusif ». L'amour est dessaisissement de soi dans un autre, qui confère au soi initial une dimension nouvelle, une plus intime relation à soi, « l'unité de la vie et de la mort au bénéfice de la vie ». Le procès spéculatif dit donc que le dieu d'Israël est un dieu relationnel. Si la phrase « Dieu est amour » est tenue pour un développement interprétatif de la phrase "je suis celui qui suis», on peut écrire «Dieu est amour », mais aussi « Dieu est amour », pour affirmer qu'il l'est véritablement. "Tu n'auras pas d'autre dieu devant ma face », dit JAHVÉ à Moïse. Peuton interpréter cette injonction comme une marque d'amour? On le peut sans doute, mais doit-on cette conclusion à l'herméneutique?

9 Un texte peut-il ainsi s'emporter vers son auteur? L'argument ontologique ne peut sortir du cercle de sa logique. Ce cercle peut poser la nécessité de l'être parfait, mais pas plus que sa nécessité, et non pas cet être lui-même. Il en est ainsi du texte biblique qui est censé être la parole de Dieu et permet ainsi de construire le projet de ce Dieu, mais non pas de voir que c'est bien un Dieu réel qui se trouve dans cette parole. Il y a un cercle herméneutique et la méthode qui permet de retrouver le sens et la portée du texte se heurte à ce fait qu'il s'agira toujours d'un texte et que nos assurances ou nos connaissances quant à l'auteur du texte viennent d'ailleurs. Ce n'est pas le texte de ses discours qui peut m'assurer de l'existence de Robespierre. L'enracinement ontologique de l'herméneutique m'ouvre bien à la reprise d'un projet de l'autre, mais pas à cet autre même, qui est sans doute au bord du texte, mais de l'autre côté. Le risque pris par Ricœur à vouloir dépasser l'alternative entre la méthode et l'ouverture en faisant de l'herméneutique un moyen de sortie de soi est de transformer la méthode en une voie de passage vers un monde avec lequel certes nous entrons en contact, mais qui induit l'intensification et la spiritualisation du procédé herméneutique. 